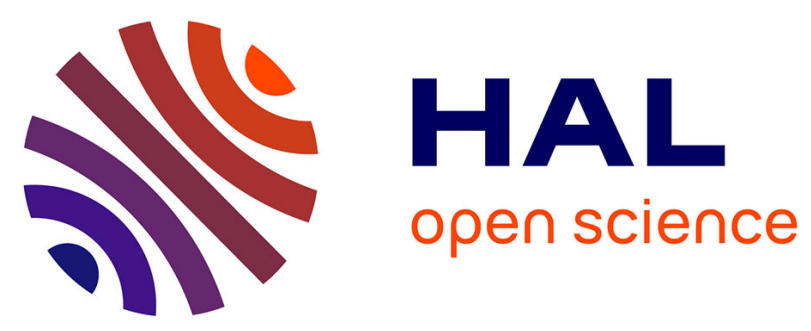

\title{
Rashba and Dresselhaus Couplings in Halide Perovskites: Accomplishments and Opportunities for Spintronics and Spin-Orbitronics
}

Mikael Kepenekian, Jacky Even

\section{> To cite this version:}

Mikael Kepenekian, Jacky Even. Rashba and Dresselhaus Couplings in Halide Perovskites: Accomplishments and Opportunities for Spintronics and Spin-Orbitronics. Journal of Physical Chemistry Letters, 2017, 8 (14), pp.3362-3370. 10.1021/acs.jpclett.7b01015 . hal-01551094

\author{
HAL Id: hal-01551094 \\ https://hal.science/hal-01551094
}

Submitted on 5 Dec 2017

HAL is a multi-disciplinary open access archive for the deposit and dissemination of scientific research documents, whether they are published or not. The documents may come from teaching and research institutions in France or abroad, or from public or private research centers.
L'archive ouverte pluridisciplinaire HAL, est destinée au dépôt et à la diffusion de documents scientifiques de niveau recherche, publiés ou non, émanant des établissements d'enseignement et de recherche français ou étrangers, des laboratoires publics ou privés. 


\title{
Rashba and Dresselhaus Couplings in Halide
}

\section{Perovskites: Accomplishments and Opportunities}

\section{for Spintronics and Spin-orbitronics}

\author{
Mikaël Kepenekian*,† and Jacky Even*,‡ \\ Institut des Sciences Chimiques de Rennes, UMR 6226, CNRS - Université de Rennes 1, France, \\ and Fonctions Optiques pour les Technologies de l'Information (FOTON), INSA de Rennes, \\ CNRS, UMR 6082, France \\ E-mail: mikael.kepenekian@univ-rennes1.fr; jacky.even@insa-rennes.fr
}

\footnotetext{
*To whom correspondence should be addressed

†'Institut des Sciences Chimiques de Rennes, UMR 6226, CNRS - Université de Rennes 1, France

${ }^{\ddagger}$ Fonctions Optiques pour les Technologies de l'Information (FOTON), INSA de Rennes, CNRS, UMR 6082, 35708 Rennes, France
} 


\begin{abstract}
In halide hybrid organic-inorganic perovskites (HOP), spin-orbit coupling (SOC) presents a well-documented large influence on band structure. However, SOC may also present more exotic effects, such as Rashba and Dresselhaus couplings. In this perspective, we start by recalling the main features of this effect and what makes HOP materials ideal candidates for the generation and tuning of spin-states. Then, we detail the main spectroscopy techniques able to characterize these effects and their application to HOP. Finally, we discuss potential applications in spintronics and in spin-orbitronics in those non-magnetic systems, that would complete the skill set of HOP and perpetuate its ride on the crest of the wave of popularity started with optoelectronics and photovoltaics.
\end{abstract}

\title{
Graphical TOC Entry
}

29

30

31

32

33

34

35

36

37

38

39

40

41

42

43

44

45

46

47

48

49

50

51

52

53

54

55

56 
The continuous success of halide hybrid organic-inorganic perovskites (HOP) as solar cell active materials follows several paths. The main activity after 2012 consisted in the search for improvement of the power conversion efficiency (PCE) in typical laboratory configuration (small area, controlled environment). It led to an unprecedented growth of the PCE that has repeatedly reached record values over 22\%. ${ }^{1,2}$ Meanwhile, another branch of HOP studies have been focused on improving the stability of those cells unfortunately sensitive to light and moisture. ${ }^{3,4}$ Opting for various mixtures of halide and cations, ${ }^{2}$ various forms, e.g. layered $\mathrm{HOP},{ }^{5-7}$ or by encapsulating the materials, the stability went from few hours to several thousands hours. Encouraged by these breakthroughs, a third path has pushed the area of hight performance solar cells from less than $1 \mathrm{~cm}^{2}$ up to $50 \mathrm{~cm}^{2}$ with a PCE of $12.6 \%,{ }^{8}$ bringing the perovskite solar cells even closer to commercial use.

A common point to all these record HOP materials is the presence of lead. Even though a large effort has been and still is dedicated to the search for efficient lead-free HOP materials, ${ }^{9,10}$ their current record PCE remains around 6.4\%. ${ }^{11}$ It is certainly unfortunate for solar cells because of the known toxicity of lead, ${ }^{12}$ even though solutions have early been proposed, ${ }^{13,14}$ however, due to the large spin-orbit coupling ( $\mathrm{SOC}$ ) exhibited by $\mathrm{Pb}$, it represents a great opportunity for optoelectronics, spintronics and spin-orbitronics applications where undesired heavy elements can find a place (e.g. arsenic, cadmium).

The first identified effect of SOC in HOP materials has been a surprising giant splitting of conduction bands, ${ }^{15,16}$ instead of the usual valence band splitting observed in classical semiconductors. ${ }^{17}$ It has a strong influence on the band gap energy as well as on the effective masses and the oscillator strength of the optical transitions at the band gap. The change of the symmetry of the bottom of the conduction band with SOC has also more subtle effects, such as a change of the selection rules for the allowed collision processes, either Auger-like effects ${ }^{18}$ or electron-phonon processes. ${ }^{19}$ In addition, Bychkov-Rashba (a.k.a. Rashba) and Dresselhaus couplings, related to the interplay between time-reversal symmetry and the lack of spatial inversion symmetry, ${ }^{20-23}$ have been predicted as early as 2013 in HOP materials by computational investigations based on 
X-ray structures. ${ }^{24-30}$ It has recently been demonstrated experimentally by angle-resolved photoemission spectroscopy (ARPES) on a single crystal of methylammonium lead bromide compound $\left(\mathrm{CH}_{3} \mathrm{NH}_{3} \mathrm{PbBr}_{3}\right),{ }^{31}$ exhibiting a coupling of the same order of magnitude than the record set by BiTeI. ${ }^{32,33}$

Several groups working in the field of halide perovskites contributed significantly in the last years toward a better understanding of their optoelectronic properties. However, various lattice instabilities and symmetry breaking are observed experimentally in this new class of semiconductors, affecting the overall performances of devices. These instabilities strongly depend on the chemical composition of the pure materials and alloys, as well as the device architecture and growth procedure. Among these instabilities, the importance of local or extended inversion symmetry breaking leading in turn to Rashba-Dresselhaus effects is still a matter of debate and deserve deeper experimental investigations.

In this perspective, we start by a brief recall of the main features of Rashba and Dresselhaus couplings as well as of the ways to characterize the effects both experimentally and computationally. We can then focus on the state-of-the-art in HOP as well as the remaining challenges in the characterization or design of the couplings in those materials. We then inspect the possible impact on photovoltaic performances. Finally, we raise the question of future applications in spintronic and spin-orbitronic devices.

\section{Main features of Rashba and Dresselhaus couplings and occurences in HOP}

A system presenting a large SOC and a lack of centrosymmetry experiences an effective magnetic field that drives a spin splitting (Figure 1-a and -b) and can lead to spin polarization even in nonmagnetic materials. ${ }^{22}$ This phenomenon has been first described by Dresselhaus in zinc-blende semiconductors, ${ }^{20}$ then by Rashba in wurtzite structures. ${ }^{34}$ Bychkov and Rashba later generalized the observation to quasi-2 dimensional systems. ${ }^{21}$ Recently, Zhang et al. have shown that spin splitting can still occurs in crystals presenting centrosymmetric bulk groups, if the site point groups do not have inversion symmetry (e.g. NaCaBi). ${ }^{35}$ 
(a)

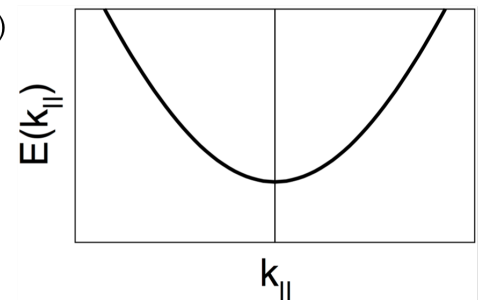

(b)

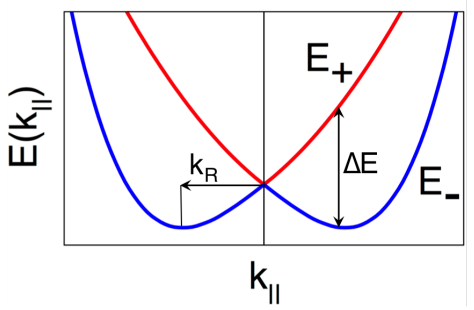

(c)

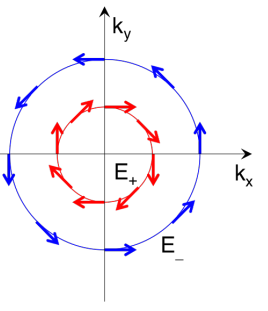

(d)

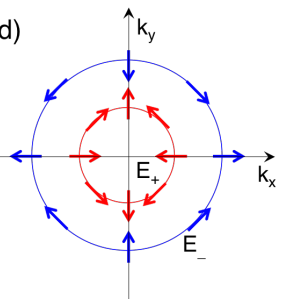

Figure 1: Schematic representation of the effect of SOC in the absence of centrosymmetry. (a) Band dispersion in the absence of SOC (b) Same including SOC. Degenerated bands now split into inner $\left(\mathrm{E}_{+}\right)$ and outer $\left(\mathrm{E}_{-}\right)$branches. Typical spin textures in the case of (c) pure Rashba, and (d) pure Dresselhaus couplings.

The strength of the effect is characterized by a parameter $\alpha$, ratio between the amplitude of the band splitting and the corresponding displacement away from the high symmetry point, $\alpha=$ $\Delta E / 2 k_{R}$

The main difference between both effects lies in the origin of the non-centrosymmetry. In the case of the Rashba effect, the asymmetry is a site inversion asymmetry (SIA), whereas the Dresselhaus effect is related to the space group of the crystal, and is often described as a bulk inversion asymmetry (BIA). Both effects can be observed separately or simultaneously and lead to typical spin textures (Figure 1-c and -d). ${ }^{22}$ A more detailed and more mathematical description of the Rashba and Dresselhaus couplings is given for instance in references 22, 29, 35, 36 and references herein. These effects have been investigated at length in classical semiconductors, either in the form of heterostructures or in the case of quantum wells. Later, examples from bulk layered materials have also exhibited large splitting, so have metal and topological insulator surfaces. These observations have already been recalled in several review articles. ${ }^{23,36-43}$

Lead- and tin-based HOP possess a natural talent for Rashba and Dresslhaus couplings with a large SOC induced by $\mathrm{Pb}^{2+}$ and $\mathrm{Sn}^{2+}$ ions and a diverse zoology of crystal structures, including many deprived of centrosymmetry. It should be pointed out, that all the reported structures for lead, 


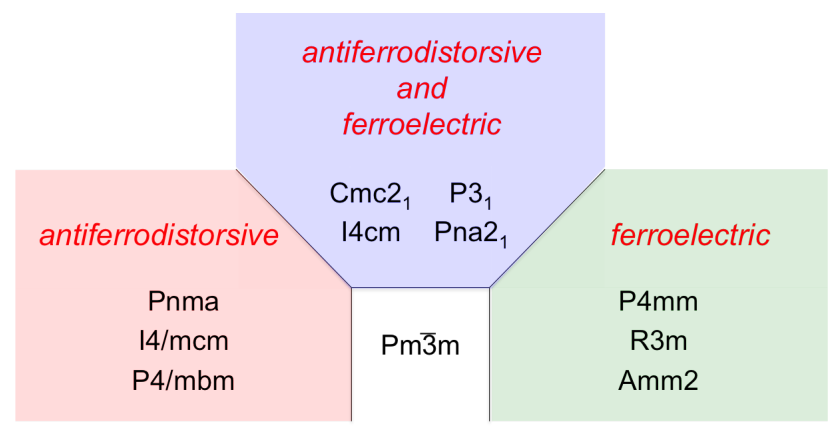

Figure 2: A few structural phase transitions observed in halide perovskites starting from the reference cubic phase $P m \overline{3} m$.

tin or germanium halide perovskites are non-magnetic leading to a time-reversal symmetry of the electronic band structure. For that reason, possible spintronic applications of halide perovskites belong to the field of spin-orbitronics. ${ }^{41}$ However, thanks to the giant SOC found in lead and tin compounds, large spin effects can be expected as soon as inversion symmetry can be broken. ${ }^{24}$ The variety of structures can be described starting from the reference phase ${ }^{9,44} P m \overline{3} m$, observed as the high temperature phase of several HOP. ${ }^{45-48}$ From this highly symmetrical structure, different phase transitions can occur being antiferrodistorsive, ferroelectric or antiferrodistorsive and ferroelectric (Figure 2). If the centrosymmetrical groups Pnma and $I 4 / \mathrm{mcm}$ have been proposed as low and room temperature structures of the notorious $\mathrm{CH}_{3} \mathrm{NH}_{3} \mathrm{PbI}_{3},{ }^{49}$ the same compound has been refined as well in the polar $P 4 \mathrm{~mm}$ group. ${ }^{9,50}$ However, the existence of a polar structure in $\mathrm{CH}_{3} \mathrm{NH}_{3} \mathrm{PbI}_{3}$ seems to contradict the classical criterion on the Goldschmidt's tolerance factor $(t>1)$ for the existence of ferroelectric phases in perovskite materials. ${ }^{51-53}$ More ferroelectric structures have been proposed for various metal, cation or halogen, among which one can find simple examples in Amm2, $R 3 m$ or $C m c 2_{1} \cdot{ }^{27,47,50,54-56}$ For 3D materials containing Ge, the tolerance factor is more compatible with the onset of a polar distortion, and a sizable SOC may also be present, especially with the contribution of heavy halogen atoms. ${ }^{57}$ More complex cases may correspond to combinations of both antiferrodistortions and ferroelectric behavior. More, due to the inherent instability of the halide perovskite lattice in most cases, local symmetry breaking may be spread in the material, at the boundaries between grains or at the surfaces.

The wealth of HOP structures available from crystallographic databases has motivated nu- 
merous computational investigations that have been the first to propose the potential occurence of Rashba and/or Dresselhaus splitting in this class of materials. ${ }^{7,24-27,29,30,58}$ The computational approach, whether it relies on density functional theory (DFT) with or without hybrid functionals, ${ }^{29,59} \mathrm{GW}$ approximation, ${ }^{60}$ or semi-empirical modeling (e.g. k $\cdot \mathbf{p}$ or tight-binding), constitutes a precious tool, combined with symmetry analysis, in the characterization of SOC-induced spin splittings. Not only is it possible to draw the dispersion of bands and thus, the band splitting away from a high symmetry point (Figure $1-\mathrm{a}$ and $-\mathrm{b}$ ), one can also plot the corresponding in-plane spin texture (Figure 1-c and -d) around the forbidden band and characterize the band splitting as a Rashba, a Dresselhaus, or a mixed Rashba/Dresselhaus splitting. In the latter case, it is then possible to assess the relative strenght of both. This has, for instance, been performed in the case of $\mathrm{CH}\left(\mathrm{NH}_{2}\right)_{2} \mathrm{SnI}_{3}$ where Rashba and Dresselhaus effects have been evaluated to $\alpha_{R}=0.50 \mathrm{eV} \cdot \AA$ and $\alpha_{D}=2.59 \mathrm{eV} \cdot \AA$, respectively. ${ }^{27,29}$

Many $\alpha$ values have been predicted on various materials. Outside of structural configurations arising from molecular dynamics (MD) calculations (vide infra), the record for a structure deduced directly from the refinement of diffraction data, is hold by the $P 4 m m$ structure of $\mathrm{CH}_{3} \mathrm{NH}_{3} \mathrm{PbI}_{3}$ with a pure Rashba coupling and a parameter $\alpha$ larger than $3.70 \mathrm{eV}$. $\mathrm{A}$ for both the valence and conduction bands. ${ }^{29}$ Before 2016, the largest value ever measured for SOC-induced couplings was $\alpha=3.80 \mathrm{eV} \cdot \AA$ for BiTeI ${ }^{32,33}$ and $\alpha=3.05 \mathrm{eV} \cdot \AA$ for $\mathrm{Bi} / \mathrm{Ag}(111) .{ }^{61}$

However, one should be careful with those values as they strongly rely on the choice made for the starting symmetry of the crystal structure, whose determination by X-ray diffraction is not always unequivocal. HOP present an additional difficulty for periodic DFT calculations. Indeed, the orientations of organic cations, e.g., $\mathrm{CH}_{3} \mathrm{NH}_{3}^{+}$affect significantly the polarization and therefore the amplitude of the splitting and they are known to undergo fast reorientations at room temperature. ${ }^{30}$ Therefore, the initial choice on the organization of organic cations impose an important bias on the results. A way around this technical issue is to substitute the dipolar cation by an inorganic cation such as $\mathrm{Na}^{+}$or $\mathrm{Cs}^{+}$. Such a strategy has been shown to preserve the band structure near the Fermi level. Snapshots of local polar structures extracted from MD simulations or 
local structure minimizations may support the idea of dynamical Rashba-Dresselhaus effects. The underlying assumption is that the crystallographic structures deduced by using classical diffraction techniques result from time averaging and do not necessarily lead to the correct prediction for carrier relaxation or recombination processes.

Among the examples of HOP expected to show a Rashba or Dresselhaus splitting, a peculiar example can be found in layered perovskites. ${ }^{7,62,63}$ Indeed, these systems are, in many ways, very similar to the heterostructures in which the Rashba and Dresselhaus effects are commonly found. In addition many examples of layered HOP are ferroelectrics. Therefore, those structures have the potential to exhibit large splittings. Indeed, several compounds belong to polar groups and have been scrutinized. ${ }^{7,29,56,64,65}$ One should notice that the above examples have a symmetry axis perpendicular to the staking direction. As a consequence, the Rashba effect is incomplete, i.e. the band splitting occur in only one direction of the Brillouin zone. ${ }^{7,29}$ Surprisingly, in the large collection of layered structures, to the best of our knowledge, none presents an absence of inversion symmetry and a $C_{2}$ axis (or higher order) in the stacking direction. Let us note that because the perovskite layers in layered structures can be non-centrosymmetric, those systems are promising structures where the hidden spin polarization proposed by Zhang et al. could be observed. ${ }^{35}$

Manifestations of spin effects in HOP are not limited to theoretical work. In 2015, a first study using pump-probe setups with circularly polarized light on thin films of $\mathrm{CH}_{3} \mathrm{NH}_{3} \mathrm{PbI}_{3}$ presented evidence for spin lifetime of about 7 ps. ${ }^{66}$ But the first direct evidence of Rashba splitting has been revealed by Niesner and coworkers who performed angle-resolved photoemission spectroscopy (ARPES) on single crystals of $\mathrm{CH}_{3} \mathrm{NH}_{3} \mathrm{PbBr}_{3} \cdot{ }^{31}$ ARPES is the most direct method to obtain information about the dispersion of the top valence bands. ${ }^{67}$ This is performed by measuring the kinetic energy and the exit angle of electrons emitted following a UV photoexcitation (Figure 3-a). In addition, spin-resolved measurements can lead to the description of spin textures. ${ }^{32,67,68}$ This straightforward technique requires however a clean single crystal sample.

Measurements on $\mathrm{CH}_{3} \mathrm{NH}_{3} \mathrm{PbBr}_{3}$ have been performed above and below the orthorhombic/cubic transition on cleaved crystals of $5 \mathrm{~mm}$, exposing the (001) surface. ${ }^{31}$ Surprisingly, at both tempera- 

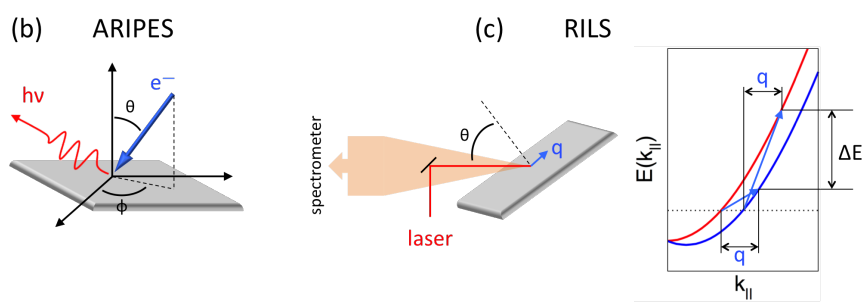

(e) Shubnikov-de Haas / WAL

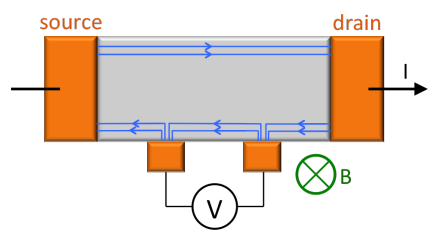

(f) CPGE

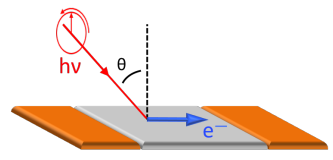

(g) SGE

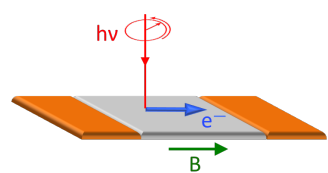

(d) pump-probe setup

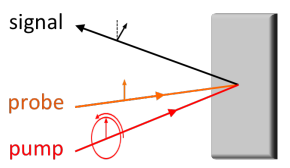

(h) MPGE

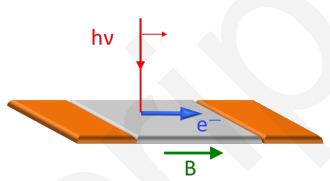

Figure 3: Schematic representation of the experimental setup of (a) ARPES and (b) ARIPES. (c) Schematic representation of RILS experiment in quasi-backscattering geometry (left) and spin-flip intrasubband transitions leading to a signal with two peaks shifted by $\Delta \mathrm{E}$ (right). The dotted line marks the position of the Fermi energy. (d) Setup for pump-probe experiments with a circularly polarized pump signal and a linearly polarized probe signal. Rashba and Dresselhaus effects are quantified by applying an additional external field being either electric or magnetic. (e) Scheme of a Hall bar for Shubnikov-de Haas or WAL studies. Oscillations in the resistivity due to edge states are monitored with respect to the amplitude of the transverse magnetic field $\mathbf{B}$ and for various gate voltages. Schemes of (f) CPGE, (g) SGE, and (h) MPGE. Photogalvanic effects rely on the spin-to-charge conversion allowed by the Rashba-Edelstein effect.

tures, a large splitting is observed with Rashba parameter $\alpha$ of $7 \pm 1 \mathrm{eV} \cdot \AA$ and $11 \pm 4 \mathrm{eV} \cdot \AA$ whereas bulk materials are expected to exhibit centrosymmetric Pnma and $P m \overline{3} m$ structures, respectively.

This is a well-known drawback of ARPES measurements: it is surface-sensitive. In this case, the loss of translational symmetry imposed by the presence of a surface solely can lead to a symmetry breaking sufficient to explain the occurence of Rashba spin splitting in these centrosymmetric groups as for instance in the case of metallic surfaces. ${ }^{36}$ One should also keep in mind that HOP are rather soft materials and tend to reorganize themselves with low energy costs. In particular, scanning tunneling microscopy (STM) performed on single crystals of $\mathrm{CH}_{3} \mathrm{NH}_{3} \mathrm{PbBr}_{3}$ has shown the presence of two surface reconstructions, which might lead to surface polar domains and therefore an enhanced splitting. ${ }^{69,70}$ Another possible explanation may rely on the strong anhamonicity of the perovskite bulk lattice, leading to large atomic motions and a dynamical effect related to polar symmetry breaking. Even all caveats taken into account, this first experimental evidence for Rashba splitting remains an important motivating force as the measured amplitude of the coupling is much larger than the previous record set by BiTeI using the same approach. Over the years, 
many spectroscopies apart from ARPES and computational approaches have been developed to assess SOC-induced splittings for surfaces and heterostructures, and are waiting to be applied to HOP. In the following, we recall some of the main spectroscopies used to unravel the presence of Rashba and Dresselhaus effects.

\section{Characterization of Rashba and Dresselhaus couplings}

ARPES is extremely powerful to describe valence states. However, HOP are characterized by a strong SOC mostly effective in the conduction bands. ${ }^{16}$ Therefore, angle-resolved inverse photoemission spectroscopy (ARIPES) would describe the part of the band structure where SOC is the most effective. In ARIPES, a beam of electron with fixed kinetic energy is used to emit photons that are detected at a fixed angle. The reciprocal space is probed by modifying the incidence angle of the electrons (Figure 3-b). ${ }^{71-73}$ As a more accurate alternative, one would turn to timeresolved-ARPES (trARPES) experiments, in which circularly-polarized pump pulses are used to access excited states. ${ }^{74}$ Chemical engineering of the Rashba-Dresselhaus effects and SOC is possible through metal substitution $(\mathrm{Pb}, \mathrm{Sn}, \mathrm{Ge})$. The ARIPES technique may provide a nice solution to probe the induced changes in the conduction band. More, the general character of the optical transition (direct versus indirect) still deserves further investigations.

In the 1990s, Jusserand and coworkers have shown that resonant inelastic light scattering (RILS) could be used to probe not only the SOC and asymmetry-induced spin splittings but also to assess the relative strength of Rashba and Dresselhaus effects. ${ }^{75,76}$ Measurements are performed in quasi-backscattering geometry (Figure 3-c) with crossed linear polarizations of the laser and scattered light (depolarized geometry). It relies on the spin-flip intrasubbands transitions between the inner and outer branches (Figure $3-c$ ). ${ }^{77}$ Thanks to the scattering angle $\Theta$, a wave vector $\mathbf{q}$ can be transferred parallel to the plane of the sample. In the presence of a spin splitting, it leads to a double peak feature related to the energy splitting at the Fermi level. Interestingly, by acting on the $n$ or $p$ doping of the sample, it has been possible to probe the splitting either in the valence or conduction band. ${ }^{75-78}$ 
However, such measurements require the Raman spectra to be conducted at very low temperature $(\mathrm{T} \approx 5 \mathrm{~K})$. In those conditions, $\mathrm{HOP}$ exhibit a stable exciton. For instance, in $\mathrm{CH}_{3} \mathrm{NH}_{3} \mathrm{PbI}_{3}$, the binding energy of the exciton at low temperature is found to be around $50 \mathrm{meV}{ }^{79}$ The interplay of the exciton with the Raman signal makes the interpretation of spectra even more delicate. Such measurements require a careful tuning of the excitation energy to avoid anomalous resonance effects occurring due to the strong resonance with excitons. ${ }^{80}$

Pump-probe optical measurements have proven to be an effective tool to investigate the spin properties of semiconductors. The absorption of circularly polarized photons initiates a population of spin-polarized, e.g. along $z$, holes or electrons. Their evolution is probed by a delayed linearly polarized pulse (Figure 3-d). The measurements therefore provide information on the longitudinal relaxation time. ${ }^{81}$

An important feature of both Rashba and Dresselhaus effects is that the combination of timereversal symmetry, SOC and the lack of inversion symmetry produces a spinor splitting, ${ }^{24}$ but not pure spin states. ${ }^{29}$ The spin continuously processes as for example in the case of a spin-valve after spin injection. ${ }^{29}$ In the usual asymmetric $2 \mathrm{D}$ electron gases, e.g. quantum wells, the dominant mechanism for spin relaxation is thus the spin precession driven by the effective magnetic field generated by the spin splitting, i.e. the D'yakonov, Perel' and Kachorovskii (DPK) mechanism, ${ }^{82,83}$ with phase changes associated to carrier scattering events as in GaAs. More, the Rashba effect in bulk HOP is linear and is thus expected to play a similar role as the Dresselhaus effect, when they are both allowed by symmetry. Additional spin relaxation mechanisms may be important for HOP, ${ }^{84}$ among them the Eliott-Yafett (EY) mechanism present in centrosymmetric semiconductors like $\mathrm{Si}^{85,86}$ In HOP, in the absence of the Rashba-Dresselhaus effects, the SOC does not split the spin-up/down states in the valence band, but the coupling with other states may nevertheless induce spin flipping as observed in the conduction band of Si. It will be of course necessary to gauge the respective importance of DPK and EY spin relaxation mechanisms in HOP. Finally let us mention the Bir-Aronov-Pikus (BAP) mechanism, ${ }^{87}$ observed for electrons in heavily p-doped classical semiconductors and related to spin-flipping associated to enhanced exchange interaction 
between electron and holes. The same situation due to reverse band ordering should affect the spins of non-equilibrium holes by exchange interactions with electrons in heavily n-doped HOP. However a strong carrier doping is only observed up to now of the p-type in tin compounds.

Several flavors of the pump-probe setup have been proposed to address specifically the case of Rashba and Dresselhaus effects occurring either separately or simultaneously. The working principle of those approaches is to modify the splitting through an external stimulus being a static electric field, ${ }^{88,89}$ a transverse static magnetic field (Hanle effect) ${ }^{90,91}$ or in transmission with an oscillating magnetic field (time-resolved Faraday rotation). ${ }^{92}$ All these alternatives are possible in HOP, since external electric and magnetic fields have already been applied with success on HOP to investigate various effects such as the influence on excitons.

A different set of experiments rely not solely on optical activities but also on the transport properties of the sample. The orignal inspections of the asymmetry induced spin splitting were performed through the analysis of the beating pattern of Shubnikov-de Haas oscillations observed thanks to a Hall bar setups (Figure 3-e). ${ }^{93,94}$ However, this approach is not deprived of ambiguities because of inhomogeneities in the concentration of carriers or of the occupation of multiple subbands.

An alternative path, using the same setup, is to take advantage of the weak antilocalization (WAL) phenomenon. At low temperature and for weak magnetic fields applied perpendicularly to the 2D gas, a magnetoresistance occurs, caused by the weak localization of electrons as a consequence of constructive interferences. The latter are suppressed by the precession of spins due to spin-orbit coupling leading to a weak antilocalization and a positive magnetoresistance. ${ }^{95}$ By varying the value of the magnetic field and the gate voltage, it is then possible to determine the strength of Rashba and Dresselhaus effects. This strategy has been used with success on various heterostructures. ${ }^{95-98}$ Its applicability to HOP will rely probably on the the growth of clean single crystal samples with well-defined facets, as well as a better understanding of the surface defects and reconstructions.

Another set of experiments giving access to the Rashba and Dresselhaus effects is based on the 
Rashba-Edelstein and inverse-Rashba-Edelstein effects, i.e. the charge-to-spin and spin-to-charge conversions. ${ }^{99,100}$ Let us mention here the circular photogalvanic effect (CPGE), the spin-galvanic effect (SGE) and the magneto-gyrotropic photogalvanic effect (MPGE). The CPGE (Figure 3f) uses a circularly polarized light to generate an unbalanced occupation in momentum space of the excited carriers because of the band splitting and spin textures induced by the Rashba and/or Dresselhaus effects. ${ }^{101-103}$ In the SGE (Figure 3-g), the electric current is caused by the out-of-equilibrium spin polarization of the system. It can be created either by the injection of a spin-current or by optical excitation. ${ }^{103-106}$ Both methods have been used in InAs and GaAs heterostructures to determine the relative amplitude of Rashba and Dresselhaus effects. ${ }^{105}$ The MPGE uses a normal incident light excitation in addition to an in-plane magnetic field to generate the photocurrent (Figure 3-h). 107,108

The application of photogalvanic effects to HOP has been theoretically examined by Li and Haney based on DFT calculations and drift-diffusion equations. ${ }^{109,110}$ One should note they used a structure exhibiting a ferroelectricity that is not predicted experimentally from the known crystallographic structures. Their investigations show that the generation of photo or spin currents are is of similar efficiency than with classical semiconductors and therefore CPGE, SGE and MPGE are promising tools to study the asymmetry-induced band splitting in HOP. Such an effect was observed in $\mathrm{CH}_{3} \mathrm{NH}_{3} \mathrm{PbI}_{3}$ as reported in a recent work from Niesner and coworkers. ${ }^{111}$ It opens a new perspective for experimental developments along this line.

\section{Effect on carrier lifetime}

Avoiding carrier recombination and thus enhancing carrier lifetime, while preserving efficient electron-hole separation after light excitation, is a key factor for optimal photovoltaic performances, especially reaching very high open circuit voltages $\left(V_{o c}\right) .{ }^{112}$ When a Rashba or Dresselhaus effect is observed on the valence and/or conduction band one can expect effect on the lifetime of the carriers. Indeed, it has been proposed that the band splitting resulting of these effects would enhance the lifetime of charge carriers because of spin-forbidden transitions ${ }^{113}$ and the resulting 
indirect band gap. ${ }^{114,115}$

The Rashba-Dresselhaus effect was proposed initially for hybrid perovskites on the basis of refined crystallographic structures exhibiting an interplay between lack of inversion symmetry, a strong SOC and time reversal symmetry. ${ }^{24-29}$ More recently, it was proposed that the centrosymmetry breaking may rather occur on a local scale through dynamical fluctuations of the structure. ${ }^{30,113-116}$ Hence, the Rashba-induced mechanism preventing carrier recombination is suspected to occurs also in structures with centrosymmetry, e.g. the room-temperature phase of $\mathrm{CH}_{3} \mathrm{NH}_{3} \mathrm{PbI}_{3}$. These theoretical propositions are based on the Born-Oppenheimer approximation and electronic band dispersions computed within a classical Brillouin zone representation for snapshots of local structures obtained by DFT-based molecular dynamics and structural minimizations. These dynamical effects are expected to have a direct impact on the effective carrier lifetime and mobility, and in a schematic picture are related to the coexistence of an indirect and a direct optical transitions. The Born-Oppenheimer approximation is justified on account of the observed timescales for the corresponding structural fluctuations, but more detailed atomistic simulations should address in the future the local nature of the lattice distortions and their impact on the local electronic density, as well as the anharmonicity of atomic motions and their impact on the carrier processes. ${ }^{115}$ This would help discussing properly the propositions related to dynamical Rashba effects with more classical propositions dealing with localizations of carriers on shallow defect states. ${ }^{117}$

\section{Applications in spintronics and spin-orbitronics}

Systems ruled by Rashba and Dresselhaus effects have been used to design devices able to (i) generate a spin-current, (ii) detect a spin-current, and (iii) modulate a spin-current. ${ }^{41,42}$ The generation of a pure spin-current, i.e. the transport of spins but not of charges, known as the spin Hall effect (SHE) has been extensively studied. ${ }^{106,118}$ In addition to the extrinsic mechanism, an intrinsic mechanism rely on the charge-to-spin conversion allowed by the Rashba-Edelstein effect. ${ }^{99,119}$ In the case of a Rashba coupling, spins are oriented perpendicularly to the momentum. 
When an electric field is applied, e.g. along $x$, the Fermi surface is displaced and electrons experienced a modified effective magnetic field which tilts the spins, creating a spin-current along $y$ (Figure 4-a). The SHE has been one of the main target of spintronics, with the notable realization of a all-semiconductor SHE transistor. ${ }^{120}$ The practical realization of similar devices in HOP will depend on the quality and processability of crystal samples, as well as the stability of the spinor-states. Deep analyses of the possible spin relaxation mechanisms mentioned before are thus required. The reciprocal mechanism that converts spin-current into charge-current is known as the

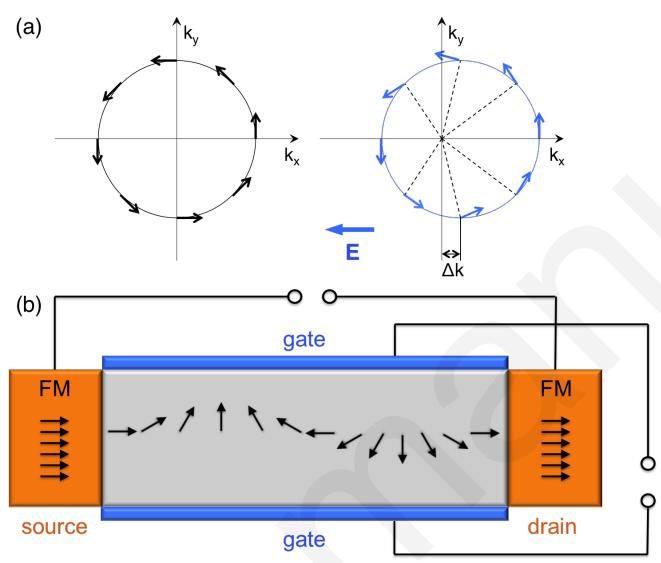

Figure 4: (a) Scheme of the Rashba-Edelstein effect. In the presence of a Rashba coupling, spins are oriented perpendicularly to the momentum (left). When an electric field is applied along $x$ (right), the Fermi surface is displaced by $\Delta \mathrm{k}$ and spins tilt, creating a spin current along $y$. (b) Scheme of a Datta-Das spin-FET. The gate voltage controls the precession of spins between the source and the drain by tuning the amplitude of the Rashba coupling.

inverse Rashba-Edelstein effect ${ }^{100}$ and is responsible for the SGE (Figure 3-g). The injection of a spin-current generates a charge current that can be detected. This phenomenon completes the toolbox necessary for spintronic devices (from charge to spin back to charge). It has recently been achieved using perovskite oxides. ${ }^{121}$

Because, Rashba and Dresselhaus couplings are sensitive to external electric fields, they can be used to manipulate spins. It has been proposed to use the electric control of spins to build a Stern-Gerlach spin-filter without ferromagnetic materials. ${ }^{122}$ An important contribution has been delivered by Datta and Das, who proposed to use this property to built a spin field effect transistor (spin-FET, Figure 4-b). ${ }^{123}$ Because spins precess under the influence of the effective magnetic 
field induced by the Rashba/Dresselhaus effects, and because their amplitude can be tuned by a gate voltage, one can control the pace of the spin precession and finally control the orientation of the spin when it reaches drain. ${ }^{124}$ The electric control over Rashba effect in the case of HOP has been proposed based on computational investigations. ${ }^{29,30}$ In the low-temperature centrosymmetric Pnma phase the Rashba coupling is naturally null, but a gate voltage of $1.25 \mathrm{~V}$ leads to a weak coupling around $\alpha=0.1 \mathrm{eV} \cdot \AA$ enough to contrast with the absence of spin precession when the gate voltage is not applied. ${ }^{29}$ Leppert and coworkers predicted that in the $P 4 m m$ phase, a coupling of nearly $1 \mathrm{eV} \cdot \AA$ could be obtained with a feasible gate voltage of $4 \mathrm{~V}$ over a $200 \mathrm{~nm}$ thick thin film. ${ }^{30}$ A limiting factor for the design of spin-FET is the spin relaxation that should allow the spin to travel from one end to the other of the device. At the time we are writing this perspective, only one measurement of spin lifetime has been reported. Pump-probe experiments have been performed on polycristalline thin films resulting in a spin relaxation lifetime of around 7 ps for electrons and nearly 1 ps for holes. ${ }^{66}$ As suggested by the authors, improvement over the quality of the sample would greatly affect these figures. Let us conclude on the possibility to manipulate individual spins, not with magnetic fields, but with gigahertz electric fields. Setups have been designed in architectures of GaAs/AlGaAs and InAs ruled by a Rashba and Dresselhaus couplings. ${ }^{125,126}$ The electric field can initialize a state, manipulate and read it. Therefore, a fully electrical manipulation of spin qubits is achieved. This possibility would require for example further investigations of spin-effects in HOP nanostructures. Available information on this topic are indeed scarce up to now in the available literature.

\section{Conclusion and perspectives}

In this perspective, we have recalled the main features of spin-orbit effects in non-centrosymmetric structures, a.k.a. Rashba and Dresselhaus effects, and explained why the observation and the engineering of these effects could be particularly attractive in HOP. After several computational works predicting the occurrence of such effects in HOP, not only has clear experimental evidence been provided, but $\mathrm{CH}_{3} \mathrm{NH}_{3} \mathrm{PbBr}_{3}$ now detains the record for highest Rashba parameter ever measured. 
This first experimental result and the recent progress in HOP crystal growth should greatly motivate complementary experimental works, whether for the characterization of couplings or their exploitation for devices. After having raised the interest of optoelectronics and microelectronics 30 years ago, and being the rising star of photovoltaics, HOP could be on the verge of making its great entrance in spintronics and spin-orbitronics.

\section{Acknowledgement}

J. E. is supported by Agence Nationale pour la Recherche (SNAP and SuperSansPlomb projects). M. K. is supported by Agence Nationale pour la Recherche (TRANSHYPERO project).

\section{References}

(1) Li, X.; Bi, D.; Yi, C.; Décoppet, J.-D.; Luo, J.; Zakeeruddin, S. M.; Hagfeldt, A.; Grätzel, M. A Vacuum Flash-assisted Solution Process for High-efficiency Large-area Perovskite Solar Cells. Science 2016, 353, 58-62.

(2) Saliba, M.; Matsui, T.; Domanski, K.; Seo, J.-Y.; Ummadisingu, A.; Zakeeruddin, S. M.; CorreaBaena, J.-P.; Tress, W. R.; Abate, A.; Hagfeldt, A.; Grätzel, M. Incorporation of Rubidium Cations into Perovskite Solar Cells Improves Photovoltaic Performance. Science 2016, 354, 206-209.

(3) Manser, J. S.; Saidaminov, M. I.; Christians, J. A.; Bakr, O. M.; Kamat, P. V. Making and Breaking of Lead Halide Perovskites. Acc. Chem. Res. 2016, 49, 330-338.

(4) Huang, W.; Manser, J. S.; Kamat, P. V.; Ptasinka, S. Evolution of Chemical Composition, Morphology, and Photovoltaic Efficiency of $\mathrm{CH}_{3} \mathrm{NH}_{3} \mathrm{PbI}_{3}$ Perovskite under Ambient Conditions. Chem. Mater. 2016, 28, 303-311.

(5) Smith, I. C.; Hoke, E. T.; Solis-Ibarra, D.; McGehee, M. D.; Karunadasa, H. I. A Layered Hybrid Perovskite Solar-Cell Absorber with Enhanced Moisture Stability. Angew. Chem. Int. Ed. 2014, 53, $11232-11235$. 
(6) Tsai, H. et al. High-efficiency Two-dimensional Ruddlesden-Popper Perovskite Solar Cells. Nature 2016, 536, 312-317.

(7) Pedesseau, L.; Sapori, D.; Traore, B.; Robles, R.; Fang, H.-H.; Loi, M. A.; Tsai, H.; Nie, W.; Blancon, J.-C.; Neukirch, A. J.; Tretiak, S.; Mohite, A. D.; Katan, C.; Even, J.; Kepenekian, M. Advances and Promises of Layered Halide Hybrid Perovskite Semiconductors. ACS Nano 2016, 10, 9776-9786.

(8) Agresti, A.; Pescetelli, S.; Palma, A. L.; Del Rio Castillo, A.; Konios, D.; Kakavelakis, G.; Razza, S.; Cinà, L.; Kymakis, E.; Bonaccorso, F.; Di Carlo, A. Graphene Interface Engineering for Perovskite Solar Modules: 12.6\% Power Conversion Efficiency over $50 \mathrm{~cm}^{2}$ Active Area. ACS Energv Lett. 2017, 2, 279-287.

(9) Stoumpos, C. C.; Kanatzidis, M. G. The Renaissance of Halide Perovskites and Their Evolution as Emerging Semiconductors. Acc. Chem. Res. 2015, 48, 2791-2802.

(10) Giustino, F.; Snaith, H. J. Toward Lead-Free Perovskite Solar Cells. ACS Energv Lett. 2016, 1, $1233-$ 1240.

(11) Noel, N. K.; Stranks, S. D.; Abate, A.; Wehrenfennig, C.; Guarnera, S.; Haghighirad, A.-A.; Sadhanala, A.; Eperon, G. E.; Pathak, S. K.; Johnston, M. B.; Petrozza, A.; Herz, L. M.; Snaith, H. J. Lead-free Organic-inorganic Tin Halide Perovskites For Photovoltaic Applications. Energy Environ. Sci. 2014, 7, 3061-3068.

(12) Babayigit, A.; Ethirajan, A.; Muller, M.; Conings, B. Toxicity of Organometal Halide Perovskite Solar Cells. Nature Mater. 2016, 15, 247-251.

(13) Chen, P.-Y.; Qi, J.; Klug, M. T.; Dang, X.; Hammond, P. T.; Belcher, A. M. Environmentally Responsible Fabrication of Efficient Perovskite Solar Cells From Recycled Car Batteries. Energv Environ. Sci. 2014, 7, 3659-3665.

(14) Chen, P.-Y.; Qi, J.; Klug, M. T.; Dang, X.; Hammond, P. T.; Belcher, A. M. Response to the Comments on "Environmentally Responsible Fabrication of Efficient Perovskite Solar Cells From Recycled Car Batteries” by Po-Yen Chen, Jifa Qi, Matthew T. Klug, Xiangnan Dang, Paula T. Hammond, and 
Angela M. Belcher Published in Energy Environ. Sci. in 2014. Energy Environ. Sci. 2015, 8, 16181625 .

(15) Even, J.; Pedesseau, L.; Dupertuis, M.-A.; Jancu, J.-M.; Katan, C. Electronic Model for Selfassembled Hybrid Organic/Perovskite Semiconductors: Reverse Band Edge Electronic States Ordering and Spin-orbit Coupling. Phvs. Rev. B 2012, 86, 205301.

(16) Even, J.; Pedesseau, L.; Jancu, J.-M.; Katan, C. Importance of Spin-Orbit Coupling in Hybrid Organic/Inorganic Perovskites for Photovoltaic Applications. J.Phvs. Chem. Lett. 2013, 4, 2999-3005.

(17) Yu, P.; Cardona, M. Fundamentals of Semiconductors: Springer, 2005.

(18) Even, J.; Pedesseau, L.; Katan, C.; Kepenekian, M.; Lauret, J.-S.; Sapori, D.; Deleporte, E. SolidState Physics Perspective on Hybrid Perovskite Semiconductors. J. Phvs. Chem. C 2015, 119, 1016110177.

(19) Nie, W.; Blancon, J.-C.; Neukirch, A. J.; Appavoo, K.; Tsai, H.; Chhowalla, M.; Alam, M. A.; Sfeir, M. Y.; Katan, C.; Even, J.; Tretiak, S.; Crochet, J. J.; Gupta, A.; Mohite, A. D. Light-activated Photocurrent Degradation and Self-healing in Perovskite Solar Cells. Nat. Commun. 2016, 7, 11574.

(20) Dresselhaus, G. Spin-Orbit Coupling Effects in Zinc Blende Structures. Phvs.Rev 1955, 100, 580586.

(21) Bychkov, Y. A.; Rashba, E. I. Properties of a 2D Electron Gas With Lifted Spectral Degeneracy. JETP Lett. 1984, 39, 78-81.

(22) Winkler, R. Spin-Orbit Coupling Effects in Two-Dimensional Electron and Hole Svstems: Springer, 2003.

(23) Bihlmayer, G.; Rader, O.; Winkler, R. Focus on the Rashba effect. New J.Phvs. 2015, 17, 050202.

(24) Even, J.; Pedesseau, L.; Jancu, J.-M.; Katan, C. DFT and k.p Modelling of the Phase Transitions of Lead and Tin Halide Perovskites for Photovoltaic Cells. Phvs. Status Solidi RRL 2014, 8, 31-35. 
(25) Brivio, F.; Butler, K. T.; Walsh, A.; van Schilfgaarde, M. Relativistic Quasiparticle Self-consistent Electronic Structure of Hybrid Halide Perovskite Photovoltaic Absorbers. Phvs. Rev. B 2014, 89, 155204.

(26) Kim, M.; Im, J.; Freeman, A. J.; Ihm, J.; Jin, H. Switchable S = 1/2 and J = 1/2 Rashba Bands in Ferroelectric Halide Perovskites. Proc. Natl. Acad. Sci. U.S.A. 2014, 111, 6900-6904.

(27) Stroppa, A.; Di Sante, D.; Barone, P.; Bokdam, M.; Kresse, G.; Franchini, C.; Whangbo, M.-H.; Picozzi, S. Tunable Ferroelectric Polarization and its Interplay with Spin-orbit Coupling in Tin Iodide Perovskites. Nature Commun. 2014, 5, 5900.

(28) Amat, A.; Mosconi, E.; Ronca, E.; Quarti, C.; Umari, P.; Nazeeruddin, M. K.; Grätzel, M.; De Angelis, F. Cation-Induced Band-Gap Tuning in Organohalide Perovskites: Interplay of Spin-Orbit Coupling and Octahedra Tilting. Nano Lett. 2014, 14, 3608-3616.

(29) Kepenekian, M.; Robles, R.; Katan, C.; Sapori, D.; Pedesseau, L.; Even, J. Rashba and Dresselhaus Effects in Hybrid Organic-Inorganic Perovskites: From Basics to Devices. ACS Nano 2015, 12, $11557-11567$.

(30) Leppert, L.; Reyes-Lillo, S. E.; Neaton, J. B. Electric Field- and Strain-Induced Rashba Effect in Hybrid Halide Perovskites. J.Phvs. Chem. Lett. 2016, 7, 3683-3689.

(31) Niesner, D.; Wilhelm, M.; Levchuk, I.; Osvet, A.; Shrestha, S.; Batentschuk, M.; Brabec, C.; Fauster, T. Giant Rashba Splitting in $\mathrm{CH}_{3} \mathrm{NH}_{3} \mathrm{PbBr}_{3}$ Organic-Inorganic Perovskite. Phvs. Rev. Lett. 2016, $117,126401$.

(32) Ishizaka, K. et al. Giant Rashba-type Spin Splitting in Bulk BiTeI. Nature Mater 2011, 10, 521-526.

(33) Eremeev, S. V.; Rusinov, I. P.; Nechaev, I. A.; Chulkov, E. V. Rashba Split Surface States in BiTeBr. New J. Phys. 2013, 15, 075015.

(34) Rashba, E. I. Properties of Semiconductors With an Extremum Loop. 1. Cyclotron and Combinational Resonance in a Magnetic Field Perpendicular to the Plane of the Loop. Sov. Phys. Solid State 1960, $2,1224-1238$. 
(35) Zhang, X.; Liu, Q.; Luo, J.-W.; Freeman, A. J.; Zunger, A. Hidden Spin Polarization in Inversionsymmetric Bulk Crystals. Nature Phvs. 2014, 10, 387-393.

(36) Krasovskii, E. E. Spin-orbit Coupling at Surfaces and 2D Materials. J.Phvs.: Condens. Matter 2015, $27,493001$.

(37) Jansen, R. Silicon Spintronics. Nature Mater 2012, 11, 400-408.

(38) Di Sante, D.; Barone, P.; Bertacco, R.; Picozzi, S. Electric Control of the Giant Rashba Effect in Bulk GeTe. Adv. Mater. 2013, 25, 509.

(39) Schliemann, J. Colloquium: Persistent Spin Textures in Semiconductor Nanostructures. $\underline{\text { Rev. Mod. }}$ Phvs. 2017, 89, 011001.

(40) Zutić, I.; Fabian, J.; Das Sarma, S. Spintronics: Fundamentals and Applications. Rev.Mod. Phvs. 2004, 76, 323-410.

(41) Manchon, A.; Koo, H. C.; Nitta, J.; Frolov, S. M.; Duine, R. A. New Perspectives for Rashba Spinorbit Couplings. Nature Mater. 2015, 14, 871-882.

(42) Soumyanarayanan, A.; Reyren, N.; Fert, A.; Panagopoulos, C. Emergent Phenomena Induced by Spin-orbit Coupling at Surfaces and Interfaces. Nature 2016, 539, 509-517.

(43) Bahramy, M. S.; Ogawa, N. Bulk Rashba Semiconductors and Related Quantum Phenomena. Adv. Mater. 2017, 10.1002/adma.201605911.

(44) Even, J. Pedestrian Guide to Symmetry Properties of the Reference Cubic Structure of 3D AllInorganic and Hybrid Perovskites. L.Phvs.Chem.LLett. 2015, 6, 2238-2242.

(45) Poglitsch, A.; Weber, D. Dynamic Disorder in Methylammoniumtrihalogenoplumbates (II) Observed by Millimeter-wave Spectroscopy. J.Chem. Phvs. 1987, 87, 6373-6378.

(46) Yamada, K.; Kuranaga, Y.; Ueda, K.; Goto, S.; Okuda, T.; Furukawa, Y. Phase Transition and Electric Conductivity of $\mathrm{ASnCl}_{3}\left(\mathrm{~A}=\mathrm{Cs}\right.$ and $\left.\mathrm{CH}_{3} \mathrm{NH}_{3}\right)$. Bull. Chem. Soc. Jpn. 1998, 71, 127-134. 
(47) Yamada, K.; Mikawa, K.; Okuda, T.; Knight, K. S. Static and Dynamic Structures of $\mathrm{CD}_{3} \mathrm{ND}_{3} \mathrm{GeCl}_{3}$ Studied by TOF High Resolution Neutron Powder Diffraction and Solid State NMR. Dalton Trans. 2002, 2112-2118.

(48) Chi, L.; Swainson, I.; Cranswick, L.; Her, J.-H.; Stephens, P.; Knop, O. The Ordered Phase of Methylammonium Lead Chloride $\mathrm{CH}_{3} \mathrm{ND}_{3} \mathrm{PbCl}_{3}$. J. Solid State Chem. 2005, 178, 1376-1385.

(49) Chen, T.; Foley, B. J.; Ipek, B.; Tyagi, M.; Copley, J. R. D.; Brown, C. M.; Choi, J. J.; Lee, S.-H. Rotational Dynamics of Organic Cations in the $\mathrm{CH}_{3} \mathrm{NH}_{3} \mathrm{PbI}_{3}$ Perovskites. Phvs. Chem. Chem. Phvs. 2015, 17, 31278-31286.

(50) Stoumpos, C. C.; Frazer, L.; Clark, D. J.; Kim, Y. S.; Rhim, S. H.; Freeman, A. J.; Ketterson, J. B.; Jang, J. I.; Kanatzidis, M. G. Hybrid Germanium Iodide Perovskite Semiconductors: Active Lone Pairs, Structural Distortions, Direct and Indirect Energy Gaps, and Strong Nonlinear Optical Properties. J.Am. Chem. Soc. 2015, 137, 6804-6819.

(51) Goldschmidt, V. M. Die Gesetze der Krystallochemie. Naturwissenschaften 1926, 14, 477-485.

(52) Kieslich, G.; Sun, S.; Cheetham, A. K. Solid-state Principles Applied to Organic ÃćÂĂÂA̧Sinorganic Perovskites: New Tricks For an Old Dog. Chem. Sci. 2014, 5, 4712-4715.

(53) Correa-Baena, J.-P.; Abate, A.; Saliba, M.; Tress, W.; Jacobsson, T. J.; Grätzel, M.; Hagfeldt, A. The Rapid Evolution of Highly Efficient Perovskite Solar Cells. Energv Environ. Sci. 2017, 10, 710-727.

(54) Stoumpos, C. C.; Malliakas, C. D.; Kanatzidis, M. G. Semiconducting Tin and Lead Iodide Perovskites with Organic Cations: Phase Transitions, High Mobilities, and Near-Infrared Photoluminescent Properties. Lnorg. Chem. 2013, 52, 9019-9038.

(55) Braun, M.; Frey, W. Crystal Structure of Bis(benzylammonium) Lead Tetrachloride, $\left(\mathrm{C}_{7} \mathrm{H}_{7} \mathrm{NH}_{3}\right)_{2} \mathrm{PbCl}_{4}$. Z. Kristallogr. New Crvst. Struct. 1999, 214, 331-332.

(56) Liao, W.-Q.; Zhang, Y.; Hu, C.-L.; Mao, J.-G.; Ye, H.-Y.; Li, P.-F.; Huang, S. D.; Xiong, R.-G. A Lead-halide Perovskite Molecular Ferroelectric Semiconductor. Nat. Commun. 2015, 6, 7338. 
(57) Katan, C.; Pedesseau, L.; Kepenekian, M.; Rolland, A.; Even, J. Interplay of Spin-orbit Coupling and Lattice Distortion in Metal Substituted 3D Tri-chloride Hybrid Perovskites. J. Mater. Chem. A 2015, 3, 9232-9240.

(58) Mosconi, E.; Umari, P.; De Angelis, F. Electronic and Optical Properties of $\mathrm{MAPbX}_{3}$ Perovskites (X = I, Br, Cl): a Unified DFT and GW Theoretical Analysis. Phvs. Chem.Chem. Phvs. 2016, 18, $27158-27164$.

(59) Demchenko, D. O.; Izyumskaya, N.; Feneberg, M.; Avrutin, V.; Özgür, U.; Goldhahn, R.; Morkosc, H. Optical Properties of the Organic-Inorganic Hybrid Perovskite $\mathrm{CH}_{3} \mathrm{NH}_{3} \mathrm{PbI}_{3}$ : Theory and Experiment. Phvs. Rev. B 2016, 94, 075206.

(60) Umari, P.; Mosconi, E.; De Angelis, F. Relativistic GW Calculations on $\mathrm{CH}_{3} \mathrm{NH}_{3} \mathrm{PbI}_{3}$ and $\mathrm{CH}_{3} \mathrm{NH}_{3} \mathrm{SnI}_{3}$ Perovskites for Solar Cell Applications. Sci.Rep. 2014, 4, 4467.

(61) Ast, C. R.; Henk, J.; Ernst, A.; Moreschini, L.; Falub, M. C.; Pacilé, D.; Bruno, P.; Kern, K.; Grioni, M. Giant Spin Splitting through Surface Alloying. Phvs. Rev. Lett. 2007, 98, 186807.

(62) Mitzi, D. B.; Chondroudis, K.; Kagan, C. R. Organic-inorganic Electronics. IBM J. Res. Dev. 2001, $45,29-45$.

(63) Saparov, B.; Mitzi, D. B. Organic-Inorganic Perovskites: Structural Versatility for Functional Materials Design. Chem. Rev. 2016, 116, 4558-4596.

(64) Daub, M.; Hillebrecht, H. Synthesis, Single-Crystal Structure and Characterization of $\left(\mathrm{CH}_{3} \mathrm{NH}_{3}\right)_{2} \mathrm{~Pb}(\mathrm{SCN})_{2} \mathrm{I}_{2}$. Angew. Chem. Int. Ed. 2015, 54, 11016-11017.

(65) Xiao, Z.; Meng, W.; Saparov, B.; Duan, H.-S.; Wang, C.; Feng, C.; Liao, W.; Ke, W.; Zhao, D.; Wang, J.; Mitzi, D. B.; Yan, Y. Photovoltaic Properties of Two-Dimensional $\left(\mathrm{CH}_{3} \mathrm{NH}_{3}\right)_{2} \mathrm{~Pb}(\mathrm{SCN})_{2} \mathrm{I}_{2}$ Perovskite: A Combined Experimental and Density Functional Theory Study. J. Phvs. Chem. Lett. 2016, 7, 1213-1218.

(66) Giovanni, D.; Ma, H.; Chua, J.; Grätzel, M.; Ramesh, R.; Mhaisalkar, S.; Mathews, N.; Sum, T. C. Highly Spin-Polarized Carrier Dynamics and Ultralarge Photoinduced Magnetization in $\mathrm{CH}_{3} \mathrm{NH}_{3} \mathrm{PbI}_{3}$ Perovskite Thin Films. Nano Lett. 2015, 15, 1553-1558. 
(67) Dil, J. H. Spin and Angle Resolved Photoemission on Non-magnetic Low-dimensional Systems. $\underline{\underline{.}}$ Phvs.: Condens. Matter 2009, 21, 403001.

(68) Santander-Syro, A. F.; Fortuna, F.; Bareille, C.; Rödel, T. C.; Landolt, G.; Plumb, N. C.; Dil, J. H.; Radović, M. Giant Spin Splitting of the Two-dimensional Electron Gas at the Surface of $\mathrm{SrTiO}_{3}$. Nature Mater. 2014, 13, 1085-1090.

(69) Ohmann, R.; Ono, L. K.; Kim, H.-S.; Lin, H.; Lee, M. V.; Li, Y.; Park, N.-G.; Qi, Y. Real-Space Imaging of the Atomic Structure of Organic-Inorganic Perovskite. J. Am. Chem. Soc. 2015, 137, 16049-16054.

(70) Murali, B. et al. Surface Restructuring of Hybrid Perovskite Crystals. ACS Energv Lett. 2016, 1, 1119-1126.

(71) Pendry, J. B. Theory of Inverse Photoemission. J. Phvs. C: Solid St. Phvs. 1981, 14, 1381-1391.

(72) König, U.; Weinberger, P.; Redinger, J.; Erschbaumer, H.; Freeman, A. J. Angle-resolved Photoemission and Inverse Photoemission From Ag(100). Phvs. Rev. B 1989, 39, 7492-7499.

(73) Sato, H.; Arita, M.; Utsumi, Y.; Mukaegawa, Y.; Sasaki, M.; Ohnishi, A.; Kitaura, M.; Namatame, H.; Taniguchi, M. Conduction-band Electronic Structure of $1 T-\mathrm{TaS}_{2}$ Revealed by Angleresolved Inverse-photoemission Spectroscopy. Phvs. Rev. B 2014, 89, 155137.

(74) Bertoni, R.; Nicholson, C. W.; Waldecker, L.; Hübener, H.; Monney, C.; De Giovannini, U.; Puppin, M.; Hoesch, M.; Springate, E.; Chapman, R. T.; Cacho, C.; Wolf, M.; Rubio, A.; Ernstorfer, R. Generation and Evolution of Spin-, Valley-, and Layer-Polarized Excited Carriers in InversionSymmetric WSe 2 . Phvs.Rev_Lett. 2016, 117, 277201.

(75) Jusserand, B.; Richards, D.; Peric, H.; Etienne, B. Zero-Magnetic-Field Spin Splitting in the GaAs Conduction Band from Raman Scattering on Modulation-Doped Quantum Wells. Phvs. Rev. Lett. 1992, 69, 848-851.

(76) Jusserand, B.; Richards, D.; Allan, G.; Priester, C.; Etienne, B. Spin Orientation at Semiconductor Heterointerfaces. Phvs. Rev. B 1995, 51, 4707(R)-4710(R). 
(77) Schönhuber, C.; Walser, M. P.; Salis, G.; Reichl, C.; Wegscheider, W.; Korn, T.; Schüller, C. Inelastic Light-scattering From Spin-density Excitations in the Regime of the Persistent Spin Helix in a GaAsAlGaAs Quantum Wells. Phvs.Rev.B 2014, 89, 085406.

(78) Korn, T.; Schüller, C. Hole Spin Dynamics and Valence-band Spin Excitations in Two-dimensional Hole Systems. Phvs. Status Solidi (b) 2014, 251, 1881-1891.

(79) D’Innocenzo, V.; Grancini, G.; Alcocer, M. J. P.; Kandada, A. R. S.; Stranks, S. D.; Lee, M. M.; Lanzani, G.; Snaith, H. J.; Petrozza, A. Excitons Versus Free Charges in Organo-lead Tri-halide Perovskites. Nat. Commun. 2014, 5, 3586.

(80) Lee, J.-U.; Park, J.; Son, Y.-W.; Cheong, H. Anomalous Excitonic Resonance Raman Effects in Fewlayered $\mathrm{MoS}_{2}$. Nanoscale 2015, 7, 3229-3236.

(81) Harley, R. T.; Karimov, O. Z.; Henini, M. Optical Control of Spins in Semiconductors. J. Phvs. D. Appl. Phvs. 2003, 36, 2198-2203.

(82) D’yakonov, M. I.; Perel', V. I. Spin Orientation of Electrons Associated With the Interband Absorption of Light in Semiconductors. Sov. Phys. JETP 1971, 33, 1053-1059.

(83) D'yakonov, M. I.; Kachorovskii, V. Y. Spin Relaxation of Two-dimensional Electrons in Noncentrosymmetric Semiconductors. Sov. Phys. Semicond. 1986, 20, 110-112.

(84) Boross, P.; Dóra, B.; Kiss, A.; Simon, F. A Unified Theory of Spin-relaxation Due to Spin-orbit Coupling in Metals and Semiconductors. Sci.Rep. 2013, 3, 3233.

(85) Elliott, R. J. Theory of the Effect of Spin-Orbit Coupling on Magnetic Resonance in Some Semiconductors. Phvs. Rev 1954, 96, 266-279.

(86) Yafet, Y. Conduction Electron Spin Relaxation in the Superconducting State. Phvs.Lett.A 1983, 98, 287-290.

(87) Bir, G. L.; Aronov, A. G.; Pikus, G. E. Spin Relaxation of Electrons Due To Scattering By Holes. Sov. Phys. JETP 1975, 42, 705-712. 
(88) Eldridge, P. S.; Leyland, W. J. H.; Lagoudakis, P. G.; Karimov, O. Z.; Henini, M.; Taylor, D.; Phillips, R. T.; Harley, R. T. All-optical Measurement of Rashba Coefficient in Quantum Wells. $\underline{P h v s .}$ Rev.B 2008, 77, 125344.

(89) Eldridge, P. S.; Leyland, W. J. H.; Lagoudakis, P. G.; Harley, R. T.; Phillips, R. T.; Winkler, R.; Henini, M.; Taylor, D. Rashba Spin-splitting of Electrons in Asymmetric Quantum Wells. Phvs. Rev. $\underline{B}$ 2010, $82,045317$.

(90) Averkiev, N. V.; Golub, L. E.; Gurevich, A. S.; Evtikhiev, V. P.; Kochereshko, V. P.; Platonov, A. V.; Shkolnik, A. S.; Efimov, Y. P. Spin-relaxation Anisotropy in Asymmetrical (001) $\mathrm{Al}_{x} \mathrm{Ga}_{1-x}$ As Quantum Wells From Hanle-effect Measurements: Relative Strengths of Rashba and Dresselhaus Spinorbit Couplings. Phvs. Rev. B 2006, 74, 033305.

(91) Eldridge, P. S.; Hübner, J.; Oertel, S.; Harley, R. T.; Henini, M.; Oestreich, M. Spin-orbit fields in Asymmetric (001)-oriented GaAs/Al $\mathrm{Ga}_{1-x}$ As Quantum Wells. Phvs. Rev. B 2011, 83, 041301(R).

(92) Meier, L.; Salis, G.; Shorubalko, I.; Gini, E.; Schön, S.; Ensslin, K. Measurement of Rashba and Dresselhaus Spin-orbit Magnetic Fields. Nature 2007, 3, 650-654.

(93) Das, B.; Miller, D. C.; Datta, S.; Reifenberger, R.; Hong, W. P.; Bhattacharya, P. K.; Singh, J.; Jaffe, M. Evidence For Spin Splitting in $\operatorname{In}_{x} \mathrm{Ga}_{1-x} \mathrm{As}_{\mathrm{s}} / \mathrm{In}_{0.52} \mathrm{qA} \mathrm{l}_{0.48} \mathrm{As}$ Heterostructures as $B \rightarrow 0$. $\underline{\text { Phvs}}$. Rev. B 1989, 39, 1411-1414.

(94) Nitta, J.; Akazaki, T.; Takayanagi, H.; Enoki, T. Gate Control of Spin-Orbit Interaction in an Inverted $\mathrm{In}_{0.53} \mathrm{Ga}_{0.47} \mathrm{As} / \mathrm{In}_{0.52} \mathrm{Al}_{0.48} \mathrm{As}$ Heterostructure. Phvs. Rev. Lett. 1997, 78, 1335-1338.

(95) Glazov, M. M.; Golub, L. E. Spin-orbit Interaction and Weak Localization in Heterostructures. Semicond. Sci. Technol. 2009, 24, 064007.

(96) Pikus, F. G.; Pikus, G. E. Conduction-band Spin Splitting and Negative Magnetoresistance in $\mathrm{A}_{3} \mathrm{~B}_{5}$ Heterostructures. Phvs. Rev. B 1995, 51, 16928-16935.

(97) Knap, W.; Skierbiszewski, C.; Zduniak, A.; Litwin-Staszewska, E.; Bertho, D.; Kobbi, F.; Robert, J. L.; Pikus, G. E.; Pikus, F. G.; Iordanskii, S. V.; Mosser, V.; Zekentes, K.; Lyanda- 
Geller, Y. B. Weak Antilocalization and Spin Precession in Quantum Wells. Phvs. Rev. B 1996, 53, 3912-3924.

(98) Miller, J. B.; Zumbühl, D. M.; Marcus, C. M.; Lyanda-Geller, Y. B.; Goldhaber-Gordon, D.; Campman, K.; Gossard, A. C. Gate-Controlled Spin-Orbit Quantum Interference Effects in Lateral Transport. Phvs. Rev. Lett. 2003, 90, 076807.

(99) Edelstein, V. M. Spin Polarization of Conduction Electrons Induced by Electric Current in TwoDimensional Asymmetric Electron Systems. Solid State Commun. 1990, 73, 233-235.

(100) Rojas-Sánchez, J. C.; Vila, L.; Desfonds, G.; Gambarelli, S.; Attané, J.-P.; De Teresa, J. M.; Magén, C.; Fert, A. Spin-to-charge Conversion Using Rashba Coupling at the Interface Between Non-magnetic Materials. Nat.Commun. 2013, 4, 2944.

(101) Ganichev, S. D.; Ivchenko, E. L.; Danilov, S. N.; Eroms, J.; Wegscheider, W.; Weiss, D.; Prettl, W. Conversion of Spin into Directed Electric Current in Quantum Wells. Phvs. Rev. Lett. 2001, 86, 43584361.

(102) Ganichev, S. D.; Ivchenko, E. L.; Prettl, W. Photogalvanic Effects in Quantum Wells. Phvsica E 2002, 14, 166-171.

(103) Ganichev, S. D.; Golub, L. E. Interplay of Rashba/Dresselhaus Spin Splittings Probed by Photogalvanic Spectroscopy - A Review. phvs. stat.sol.(b) 2014, 251, 1801-1823.

(104) Ganichev, S. D.; Ivchenko, E. L.; Bel'kov, V. V.; Tarasenko, S. A.; Sollinger, M.; Weiss, D.; Wegscheider, W.; Prettl, W. Spin-galvanic Effects. Nature 2002, 417, 153-156.

(105) Giglberger, S.; Golub, L. E.; Bel'kov, V. V.; Danilov, S. N.; Schuh, D.; Gerl, C.; Rohlfing, F.; Stahl, J.; Wegscheider, W.; Weiss, D.; Prettl, W.; Ganichev, S. D. Rashba and Dresselhaus Spin Splittings in Semiconductor Quantum Wells Measured by Spin Photocurrents. Phvs. Rev. B 2007, 75, 035327.

(106) Sinova, J.; Valenzuela, S. O.; Wunderlich, J.; Back, C. H.; Jungwirth, T. Spin Hall Effects. Rev.Mod. Phvs. 2015, 87, 1213-1259. 
(107) Bel'kov, V. V.; Olbrich, P.; Tarasenko, S. A.; Schuh, D.; Wegscheider, W.; Korn, T.; Schüller, C.; Weiss, D.; Prettl, W.; Ganichev, S. D. Symmetry and Spin Dephasing in (110)-Grown Quantum Wells. Phvs.Rev._Lett. 2008, 100, 176806.

(108) Bel'kov, V. V.; Ganichev, S. D.; Ivchenko, E. L.; Tarasenko, S. A.; Weber, W.; Giglberger, S.; Olteanu, M.; Tranitz, H.-P.; Danilov, S. N.; Schneider, P.; Wegscheider, W.; Weiss, D.; Prettl, W. Magneto-gyrotropic Photogalvanic Effects in Semiconductor Quantum Wells. J. Phvs.: Condens. Matter 2005, 17, 3405-3428.

(109) Li, J.; Haney, P. M. Optical Spintronics in Organic-inorganic Perovskite Photovoltaics. Phvs. Rev. B 2016, 93, 155432 .

(110) Li, J.; Haney, P. M. Circular Photogalvanic Effect in Organometal Halide Perovskite $\mathrm{CH}_{3} \mathrm{NH}_{3} \mathrm{PbI}_{3}$. Appl. Phvs. Lett. 2016, 109, 193903.

(111) Niesner, D.; Hauck, M.; Shrestha, S.; Levchuk, I.; Matt, G. J.; Osvet, A.; Batentschuk, M.; Brabec, C.; Weber, H. B.; Fauster, T. Spin-split Bands Cause the Indirect Band Gap of $\left(\mathrm{CH}_{3} \mathrm{NH}_{3}\right) \mathrm{PbI}_{3}$ : Experimental Evidence From Circular Photogalvanic Effect. arXiv:1703.08740v1.

(112) Polman, A.; Knight, M.; Garnett, E. C.; Ehrler, B.; Sinke, W. C. Photovoltaic materials: Present efficiencies and future challenges. Science 2016, 352, aad4424.

(113) Zheng, F.; Tan, L. Z.; Liu, S.; Rappe, A. M. Rashba Spin-Orbit Coupling Enhanced Carrier Lifetime in $\mathrm{CH}_{3} \mathrm{NH}_{3} \mathrm{PbI}_{3}$. Nano Lett. 2015, 15, 7794-7800.

(114) Azarhoosh, P.; McKechnie, S.; Frost, J. M.; Walsh, A.; van Schilfgaarde, M. Research Update: Relativistic Origin of Slow Electron-hole Recombination in Hybrid Halide Perovskite Solar Cells. $\underline{A P L}$ Mater. 2016, 4, 091501.

(115) Etienne, T.; Mosconi, E.; De Angelis, F. Dynamical Origin of the Rashba Effect in Organohalide Lead Perovskites: A Key to Suppressed Carrier Recombination in Perovskite Solar Cells? L.Phvs. Chem. Lett. 2016, 7, 1638-1645.

(116) Yu, Z.-G. Rashba Effect and Carrier Mobility in Hybrid Organic-Inorganic Perovskites. L. Phvs. Chem.Lett. 2016, 7, 3078-3083. 
(117) Kirchartz, T.; Rau, U. Decreasing Radiative Recombination Coefficients via an Indirect Band Gap in Lead Halide Perovskites. J.Phvs. Chem. Lett. 2017, 8, 1265-1271.

(118) Hoffmann, A. Spin Hall Effects in Metals. IEEE Trans. Magn. 2013, 49, 5172-5193.

(119) Sinova, J.; Culcer, D.; Niu, Q.; Sinitsyn, N. A.; Jungwirth, T.; MacDonald, A. H. Universal Intrinsic Spin Hall Effect. Phvs. Rev_Lett. 2004, 92, 126603.

(120) Wunderlich, J.; Park, B.-G.; Irvine, A. C.; Zârbo, L. P.; Rozkotová, E.; Nemec, P.; Novál, V.; Sinova, J.; Jungwirth, T. Spin Hall Effect Transistor. Science 2010, 330, 1801-1804.

(121) Lesne, E. et al. Highly Efficient and Tunable Spin-to-charge Conversion Through Rashba Coupling at Oxide Interfaces. Nature Mater. 2016, 15, 1261-1266.

(122) Ohe, J.; Yamamoto, M.; Ohtsuki, T.; Nitta, J. Mesoscopic Stern-Gerlach Spin Filter by Nonuniform Spin-orbit Interaction. Phvs. Rev.B 2005, 72, 041308(R).

(123) Datta, S.; Das, B. Electronic Analog of the Electrooptic Modulator. Appl. Phvs. Lett. 1990, 56, 665667.

(124) Koo, H. C.; Kwon, J. H.; Eom, J.; Chang, J.; Han, S. H.; Johnson, M. Control of Spin Precession in a Spin-Injected Field Effect Transistor. Science 2009, 325, 1515-1518.

(125) Nowack, K. C.; Koppens, F. H. L.; Nazarov, Y. V.; Vandersypen, L. M. K. Coherent Control of a Single Electron Spin with Electric Fields. Science 2007, 318, 1430-1433.

(126) Nadj-Perge, S.; Frolov, S. M.; Bakkers, E. P. A. M.; Kouwenhoven, L. P. Spin-orbit Qubit in a Semiconductor Nanowire. Nature 2010, 468, 1084-1087. 